\title{
Massive pre-main sequence stars in M17
}

\section{Maria C. Ramirez-Tannus ${ }^{1}$, Lex Kaper ${ }^{1}$, Bram B. Ochsendorf $^{1}$ and Lucas E. Ellerbroek ${ }^{1}$}

${ }^{1}$ Anton Pannekoek Institute for Astronomy, University of Amsterdam P.O. Box 94249, Science Park 904, 1090 GE Amsterdam, The Netherlands

email: m.c.ramireztannus@uva.nl

\begin{abstract}
We have obtained optical to near-infrared (300-2500 nm) VLT/X-shooter spectra of six candidate mYSOs, deeply embedded in the massive star forming region M17. These mYSO candidates have been identified based on their infrared excess and spectral features (doublepeaked emission lines, CO band-head emission) indicating the presence of a disk (Hanson et al. 1997). In most cases, we detect a photospheric spectrum allowing us to measure the physical properties of the mYSOs and to confirm their PMS nature.
\end{abstract}

Keywords. stars: formation, stars: pre-main-sequence, stars: massive

\section{Introduction}

The formation process of massive stars is still poorly understood. Formation timescales are short, the corresponding accretion rates very high, and the forming stars are hidden from view due to vast amounts of interstellar extinction. Evidence is accumulating that the formation process of massive stars is through disk accretion, similar to low-mass stars. This despite the physical processes acting near hot, massive stars (e.g., radiation pressure, ionization) that may reverse the accretion flow and prevent matter from accreting onto the forming star (Zinnecker \& Yorke 2007; Keto \& Wood 2006; Krumholz et al. 2009). Given the short formation time (and lifetime) of massive stars, the accretion rate must be high (up to $10^{-3} \mathrm{M}_{\odot} \mathrm{yr}^{-1}$, Hosokawa et al. 2010). At these accretion rates, the massive Young Stellar Object (mYSO) is expected to bloat up to about $100 \mathrm{R}_{\odot}$, resulting in a relatively low effective temperature and low UV luminosity. Simulations of different accretion models applied to a large sample of mYSOs suggest that the accretion rate increases as the mYSO grows in mass (Davies et al. 2011). When the accretion process has stopped, the "bloated" pre-main-sequence (PMS) star contracts to the main sequence on the Kelvin-Helmholtz timescale. Recently, such bloated, massive PMS stars have been spectroscopically confirmed (Ochsendorf et al. 2011; Ellerbroek et al. 2013).

We are now on the verge of being able to test these models quantitatively. However, the sample of mYSOs for which the photospheric spectrum has been detected is very small. We have obtained high-quality spectra with X-shooter on the ESO Very Large Telescope of $7 \mathrm{mYSOs}$ and 3 O-stars (red and blue in Fig. 1(b), respectively), first identified by Hanson et al. 1997, in the prominent Galactic H II region M17.

\section{Photospheric spectra mYSOs}

In Figure 1(a) we show the photospheric spectra of our sample of stars. For the B-type stars we observed the ratio He I $\lambda \lambda 4471 / \mathrm{Mg}$ II $\lambda \lambda 4481$, which is a good temperature indicator in late-B stars (Gray and Corbally, 2009). The hottest stars (from B0.5 and O types) were mainly classified by the presence of He II lines. We further constrained the temperature, luminosity class, projected rotational velocity, and $\log g$ of the stars by comparing the $\mathrm{H}$ and He observed absorption lines with model profiles produced by FASTWIND (Puls et al. 2005). We performed a $\chi^{2}$ test to find the best fitting model. In Figure 1(a) we show a section of the spectra including the model fits. In 5 of our objects we identify several double peaked emission lines with peakto-peak velocity separation between 70 and $120 \mathrm{~km} / \mathrm{h}$. For 3 of them (B268, B275 and B331) we identify CO-bandhead emission. 


\section{Conclusions}

Having thus obtained the stellar parameters of the mYSOs, we can place them in the HRD (Fig. 1(b)). The objects are located on PMS tracks and are contracting towards the main sequence, consistent with a "birth line" accretion rate $<10^{-4} \mathrm{M}_{\odot} \mathrm{y}^{-1}$ and age $<10^{5} \mathrm{yr}$.

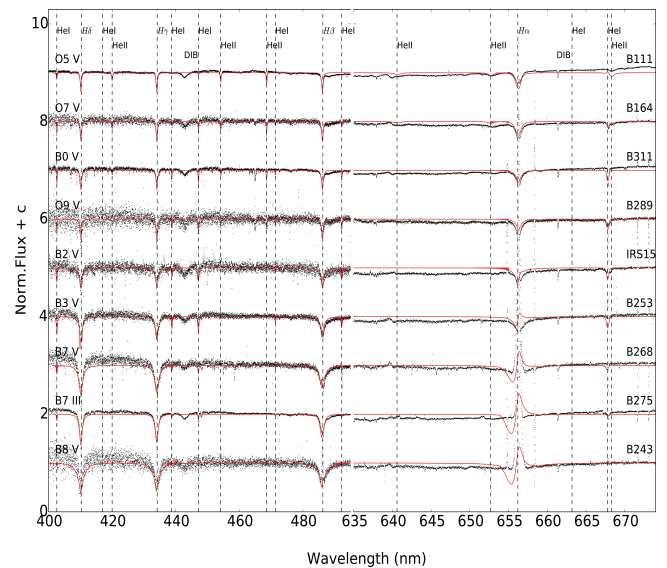

(a) Photospheric spectra.

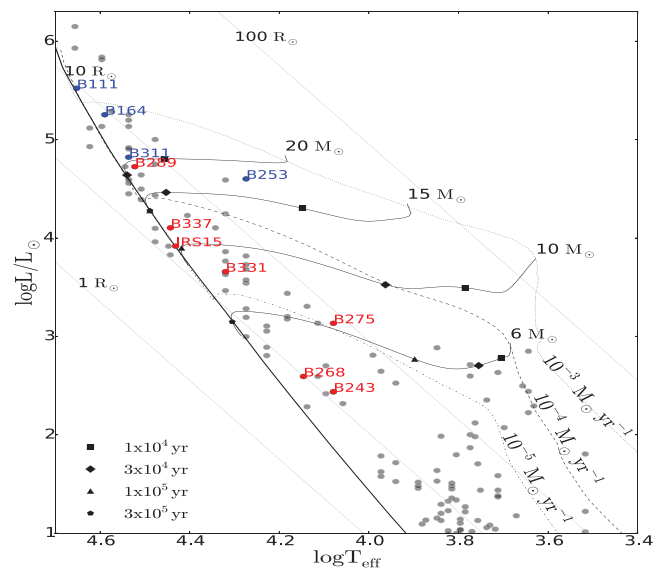

(b) Hertzprung-Russell diagram.

Figure 1. (a) Visual spectra of the M17 sample (black dots), with their corresponding FASTWIND models in red. (b) HRD of M17: location of our X-shooter targets (O stars in blue and mYSOs in red) with respect to PMS tracks from Hosokawa \& Omukai (2009) with the ZAMS mass labeled. The dashed and dot-dashed lines are birth lines corresponding to different accretion rates.

\section{References}

Davies, B., Hoare, M. G., Lumsden, S. L., et al. 2011, MNRAS, 416, 972

Ellerbroek, L. E., Bik, A., Kaper, L., et al. 2013, A\&̈A, 558, A102

Gray, R. O. \& Corbally, J., C. 2009, Stellar Spectral Classification, (Princeton University press)

Hanson, M. M., Howarth, I. D., \& Conti, P. S. 1997, ApJ, 489, 698

Hosokawa, T., Yorke, H. W., \& Omukai, K. 2010, ApJ, 721, 478

Keto, E. \& Wood, K. 2006, ApJ, 637, 850

Krumholz, M. R., Klein, R. I., McKee, C. F., et al. 2009, Science, 323, 754

Ochsendorf, B. B., Ellerbroek, L. E., Chini, R., et al. 2011, A\&3A,536, L1

Puls, J., Urbaneja, M. A., Venero, R., et al. 2005, A\&A, 435, 669

Zinnecker, H. \& Yorke, H. W. 2007, ARA\&A, 45, 481 